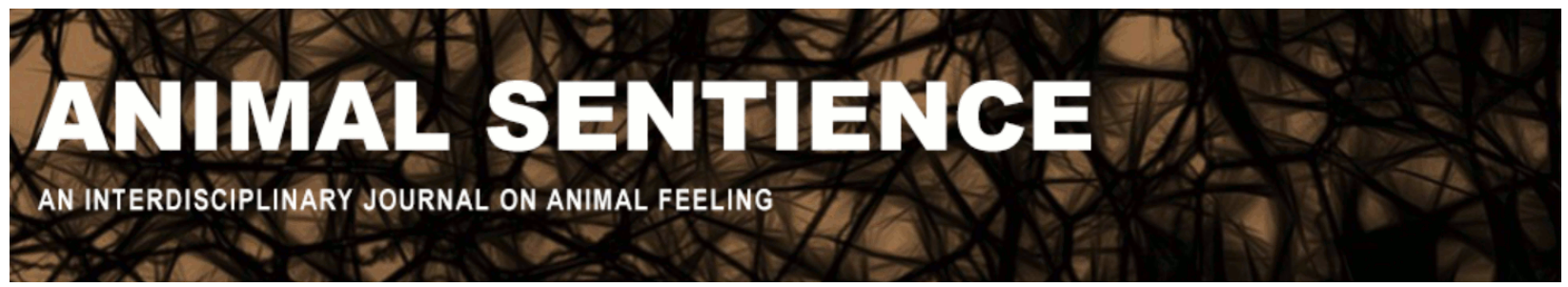

Devor, Marshall (2016) Where is pain in the brain?. Animal Sentience 3(34) DOI: 10.51291/2377-7478.1071

Date of submission: 2015-12-04

Date of acceptance: 2015-12-13

(c) (i)




\title{
Where is pain in the brain?
}

Commentary on Key on Fish Pain

\author{
Marshall Devor \\ Institute of Life Sciences \& Center for Research on Pain \\ The Hebrew University of Jerusalem
}

\begin{abstract}
Key argues that fish cannot experience pain based on (1) brain imaging in humans, (2) consequences of lesions and (3) direct brain stimulation. Imaging indeed shows that painrelevant signals reach the cortex, but not that they underlie the subjective experience of pain. Lesions and stimulation data are more to the point, but Key paints an idiosyncratic and misleading picture of their effects. S1 and S2 ablation does not eliminate evoked or spontaneous pain, although there may be up- or down-modulation. Likewise, stimulation of pain-associated cortical areas rarely induces pain, and pain almost never occurs at the onset of epileptic seizures. In contrast, cortical lesions and activation do have striking and reliable effects on visual, auditory, smell and touch perception. Overall, the case for the cerebral cortex being an essential substrate for pain experience in humans is too equivocal a starting point for ruling out the possibility of pain experience in fish.
\end{abstract}

\footnotetext{
Marshall Devor marshlu@mail.huji.a.il is Professor of Pain Research, Department of Cell \& Developmental Biology, Institute of Life Sciences, Hebrew University of Jerusalem, Israel. His major contributions have been concerning the physiological basis of neuropathic pain and more recently, the mechanisms involved in the loss of consciousness and in pain-free surgery. http://new.huji.ac.il/en/staff/9f974a003cc84fe4d0aff333b608f169
}

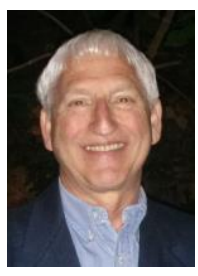

Introduction. Fish, like most members of the animal kingdom, react to noxious stimuli with adaptive, sometimes complex behavioral and physiological responses. Such nocifensive responses can also be programed into devices. They do not require, or necessarily imply, conscious perception. But to "experience" (i.e., feel) pain does. Asking whether fish, or devices, can in principle feel pain is to query the minimal requirements for conscious perception in general. The sad fact is that we have no realistic notion of how electrochemical activity in the brain yields conscious experience. "A spotlight," "a workspace," "an emergent property" are more metaphors than mechanisms. Key's (2016) call for mechanistic explanations of pain experience is right on target, but hard to deliver. We don't even know in what sense the issue is hard. Is it complex, like sequencing the genome, or is it more like Darwinian evolution ... an idea that is conceptually simple, but requires special insight to invent? Key himself deals with neural correlates of pain (vs. nociception), not biological mechanisms per se. He argues that since consciousness is mediated by the cerebral cortex in humans, and the fish brain does not have a sufficiently cortex-like structure, fish could not "feel" pain (or anything else). The logical flaw in this inference has been addressed by other commentators. Instead, I will consider Key's basic premise: how confident can we be that pain experience in humans is mediated by the cerebral cortex? 
Cortex and pain. The dogma that the cortex is the agent of all consciousness experience, including pain, is deeply seated. It originates in the fact that we humans stand out in the proportional size of our cortex, and in its complexity and the richness of its intrinsic connectivity. Some people acknowledge consciousness only in humans. Most, however, concede that primates may also have a mental life with the level of uncertainty tending to increase with decreasing cortical size in the species in question. Since we lack a clear idea of what the adaptive value of consciousness might be, we can easily imagine lower animals (and humanoid "zombies") getting along without it, in a machine-like manner. Consciousness might amount to a luxury of higher mammals, something useful for enjoying poetry and contemplating the future. Functional brain imaging in humans reveals activations in numerous presumably pain-related cortical areas, the so-called "pain-matrix" (or "-signature", or "-fingerprint") (Peyron, Laurent et al. 2000; Mouraux, Diukova et al. 2010). Activations in some matrix areas track stimulus and/or perceptual intensity, like a thermometer. But in others the signal tracks changes in evaluative aspects of pain (e.g., unpleasantness, suffering), features that seems more like perception (Rainville, Duncan et al. 1997). The degree of unpleasantness evoked by a fixed noxious stimulus can be varied experimentally using a placebo or hypnotic suggestion, among other approaches. But the fact that the cortex receives pain-related input and can track its unpleasantness does not mean that the cortex is the generator of the pain experience. Many subcortical areas are also activated by "painful stimuli" (that is, noxious stimuli that evoke a report of pain). Cortical activations could well reflect ascending drive, while cortical modulation of pain could reflect descending control.

Consider the cerebellum. Despite being "lit up" by painful stimuli in fMRI studies, cerebellar damage does not eliminate pain perception (or obtund consciousness) and cerebellar stimulation does not evoke pain. For this reason, nobody considers it the generator of conscious pain experience. The presence of cerebellar activations simply means that information about noxious events is relevant to what the cerebellum does, namely, generating complex movement synergies. The elegance of your tennis swing is very likely to be affected if, as you run to intercept the ball, you step hard on a nail. Likewise for the cortex. Many cortical functions such as memory, response planning and anticipation also require information about pain. As noted, the injection of words (viz., verbal suggestion) is enough to strongly modulate both pain experience and pain-matrix activations, and language is unquestionably a cortical function. But this in no way requires that cortical activations form the substrate for the "raw feel" of pain.

Cortical activity as a cause of pain perception: stimulation. In contrast to PET and fMRI activations, which are at best suggestive, perceptual effects of cortical stimulation and cortical ablation do speak directly to the question of whether the cortex is sufficient and/or necessary for pain perception. The overall picture here is both fairly clear and fairly counterintuitive. Direct electrical stimulation of the cerebral cortex, including in areas that show prominent activations in response to pain-provoking stimuli, almost never evokes a report of pain in awake patients (Penfield and Rasmussen 1955; Libet 1973; Mazzola, Isnard et al. 2012). The same is true of transcranial magnetic stimulation. The failure to evoke pain contrasts markedly with other sensory modalities. Stimulating the visual cortex in awake humans reliably evokes a visual 
percept, stimulating the auditory cortex evokes sound, the olfactory cortex smell and the somatosensory cortex (non-painful) touch.

Key's statement that Penfield and Rasmussen (1955) reported having evoked pain is formally correct, but it is highly disingenuous. Pain was reported in only $1 \%$ of stimulation sites, even though a large fraction of the sites tested were within pain matrix areas. Penfield himself noted his failure to find a primary pain area (P1) in the cortex and subsequent attempts confirmed this. To cushion the blow, Key adds that Penfield also had difficulty evoking "complex visual images" such as faces, obtaining such reports at "only" $5 \%$ of stimulation sites in visual areas. Actually, it is astounding that faces were ever evoked! But is this really relevant to pain? In visual areas, simple evoked percepts (light, patterns) were near universal. Crude retinal stimulation never evokes faces, whereas pain is reliably evoked by stimulation in a variety of subcortical loci, from the spinal cord through the brainstem and the thalamus (Casey 1971; Dostrovsky 2000).

Potential exceptions to the failure of cortical stimulation to elicit pain are recent reports of pain being evoked by electrical stimulation in the posterior insular cortex/dorsal operculum, mostly as part of a diagnostic protocol in patients with disabling epilepsy (Penfield and Faulk Jr. 1955; Mazzola, Isnard et al. 2006; Mazzola, Isnard et al. 2012). However, even here, pain was evoked in only a small minority of patients and sites stimulated ( 10\%). Furthermore, the result is inconsistent with observations of others who also stimulate the insular cortex in awake humans. ${ }^{1}$ It is also inconsistent with the rarity of pain as an aura of insular seizures, including in the stimulated patients themselves (Montavont, Mauguiere et al. 2015), and with the fact that major destruction of the posterior insula, by most reports, does not suppress pain sensation (Starr, Sawaki et al. 2009; Damasio, Damasio et al. 2013; Baier, zu Eulenburg et al. 2014; Davis, Bushnell et al. 2015; Feinstein, Khalsa et al. 2015). Can one be confident that stimulation-evoked pain in the few cases where this occurred was not due to subcortical propagation of neural activity? Declaring the posterior insula to be the long-sought P1 is at best premature.

If the posterior insula is not $\mathrm{P} 1$, perhaps there is some other cortical structure whose activity constitutes the neural substrate of pain experience. This structure, like the insular cortex, may be inaccessible to surface stimulation, but have no associated clinical condition that would have occasioned the insertion of depth electrodes. Even this hypothetical scenario, however, is a stretch. Epileptic seizures are a common medical event and they frequently involve buried/ inaccessible foci such as in limbic structures of the frontal and temporal lobes. Nonetheless, as noted, it is very rare for epilepsy to include auras that are painful. An ad-hoc explanation sometimes offered is that pain is unique among the senses in requiring simultaneous activation at multiple cortical sites. However, spread of seizure activity across the cortex is also fairly common and here too, painful auras are rare (Nair, Najm et al. 2001; Montavont, Mauguiere et al. 2015).

\footnotetext{
${ }^{1}$ M. S. Berger (UCSF), personal communication (2/2009), quoted with permission: “I've operated on more than 175 insular tumors, and although I have not mapped all of them, I can tell you that even when patients are awake I have never seen pain being evoked by stimulating, touching or even resecting the posterior insula."
} 
Cortical activity as a cause of pain perception: ablation. Perhaps pain is far more complex than vision, audition etc., requiring more precise orchestration of cortical activity even than face perception. This could explain why crude activation and seizures almost never evoke pain. But not only is this idea counterintuitive a priori, it does not fit with empirical observations. The requirement for precise, complex orchestration would necessarily mean that the pattern, and the resulting percept, would be fragile and easily disrupted by crude electrical, magnetic or epileptic activation, or by lesions, rendering the individual "blind" to pain, spontaneous or stimulus-evoked. In fact, lesions that involve pain matrix areas are very common (e.g., following strokes), and they are almost never accompanied by dense analgesia, widespread or focal. On the contrary, such lesions frequently trigger chronic "post-stroke pain," suggesting release from baseline suppression by the cortex (Boivie, Leijon et al. 1989; Hansen, Marcussen et al. 2012).

Over the years, occasional case reports have described strokes or trauma that reduced pain in the somatotopically corresponding limb. Key cites a few such cases, implying that this is the rule. However, this effect is highly exceptional and almost always transient. Focal cortical ablation in the primary representation of a painful limb (postcentral gyrectomy) has been tried extensively as a remedy for chronic pain and it failed resoundingly, at best providing temporary relief (White and Sweet 1969; Gybels and Sweet 1990; Roland 1992). For this reason, it is not offered today as a therapeutic option even in cases of severe, intractable pain. It simply doesn't work. In contrast, lesions of cortical areas that serve other senses do cause striking sensory deficits, notably V1 lesions that cause localized scotomas or whole-field perceptual blindness. Interestingly, blindness due to $\mathrm{V} 1$ lesions, and even much broader cortical damage, is not necessarily accompanied by loss, or even detectable attenuation of consciousness (White and Sweet 1969; Posner, Saper et al. 2007; Philippi, Feinstein et al. 2012).

Although cortical activation rarely causes pain and cortical ablation rarely yields analgesia, the cortex is deeply involved in evaluating the significance of pain (Is the baby coming? Is it cancer?). Cognitive, emotional and other psychosocial variables profoundly color pain experience and the degree of suffering attached to it. This works in both directions. The placebo effect and distraction, for example, suppress pain experience while nocebo and catastrophic thinking ("catastrophizing") augments it. As expected, surgical ablation (and perhaps cortical stimulation) can emulate such modulation. Examples of the former are prefrontal leucotomy, cingulumotomy and cingulotomy (White and Sweet 1969; Wilkinson, Davidson et al. 1999; Burchiel 2015). Chronic pain patients who have undergone such operations are reported to declare that their pain hurts as much as ever, but that they no longer care. Cortical lesions can also alter sensory quality causing tactile allodynia, or a burning-hot or freezing-cold sensation upon mild stimulation, or spontaneously (Veldhuijzen, Greenspan et al. 2010). Right parietal stroke sometimes produces the particularly bizarre symptom of sensory hemi-neglect, where the patient denies outright that his left arm or leg belongs to him. But despite this, noxious stimulation of the denied limb evokes wincing, autonomic responses, withdrawal and pain report. Pain is experienced, but its source is detached from the individual's body schema. Body schema is a cortical function, but not necessarily pain. In general, cortical modulation of pain experience should not be confused with the generation of pain. 
Evolutionary perspective and consequences for pain in fish. It takes a big cortex to determine whether contrasts sweeping across the retina represent a cloud passing by or a charging rhinoceros at 30 meters. Feature extraction in other sensory realms also requires considerable neural processing. Pain is different in that the mission-critical information required for arousal and escape is already available at the first central synapse. In this sense, pain/nociception is a primitive sense and as such, might have served as the original substrate for the evolution of conscious perception, long before heavy-duty cortical data processing came on line. In this scenario, context- and language-related modulation of pain perception, which surely does require high-level cortical processing, is a later refinement; adaptive but non-essential. More speculatively still, with the algorithm for consciousness already running in phylogenetically old subcortical structures, the evolving cortex might have begun to provide it with processed multisensory input much as the spinal cord had long been providing it with nociceptive input. This architecture may account for why dysfunction in V1 causes perceptual blindness without blunting consciousness, while dysfunction at focal subcortical loci can cause loss of consciousness encompassing all sensory modalities (Devor and Zalkind 2001; Posner, Saper et al. 2007; Minert and Devor 2015).

The cortex registers and employs information about pain, and can modulate the sensory, cognitive and affective color of pain experience. However, the empirical data do not fit well with pain experience itself arising from neural activity in the cortex. We should not let ideology blur our appreciation of the stark differences between pain and the other senses (including touch) when it comes to the consequences of cortical activation and ablation. Pain experience is clearly an outcome of brain activity, but a healthy skepticism is called for regarding the primacy of the cerebral cortex (Panksepp 2011; Damasio and Carvalho 2013). The possibility of sub-cortical activity being the primary substrate of pain experience ought to be taken seriously, even with respect to humans. Correspondingly, the possibility of pain in fish cannot be dismissed simply because they lack an adequate cortex.

\section{References}

Baier, B., zu Eulenburg, P., Geber, C., Rohde, F., Rolke, R., Maihöfner, C., Birklein, F. and Dieterich, M. (2014). Insula and sensory insular cortex and somatosensory control in patients with insular stroke. Eur J Pain 18(10): 1385-1393.

Boivie, J., Leijon, G. and Johansson, I. (1989). Central post-stroke pain-a study of the mechanisms through analysis of the sensory abnormalities. Pain 37: 173-185.

Burchiel, K. J. ( Ed.). (2015). Surgical management of pain. New York, Thieme.

Casey, K. L. (1971). Somatosensory responses of bulboreticular units in awake cat: relation to escape-producing stimuli. Science 173(3991): 77-80. 
Damasio, A. and Carvalho, G. B. (2013). The nature of feelings: evolutionary and neurobiological origins. Nat Rev Neurosci 14(2): 143-152.

Damasio, A., Damasio, H. and Tranel, D. (2013). Persistence of feelings and sentience after bilateral damage of the insula. Cereb Cortex 23(4): 833-846.

Davis, K. D., Bushnell, M. C., lannetti, G. D., St. Lawrence, K. and Coghill, R. (2015). Evidence against pain specificity in the dorsal posterior insula. F1000Res 4: 362.

Devor, M. and Zalkind, V. (2001). Reversible analgesia, atonia, and loss of consciousness on bilateral intracerebral microinjection of pentobarbital. Pain 94(1): 101-112.

Dostrovsky, J. O. (2000). Role of thalamus in pain. Prog Brain Res 129: 245-257.

Feinstein, J.S., Khalsa, S.S., Salomons, T.V., Prkachin, K.M., Frey-Law, L.A., Lee, J.E., Tranel, D. and Rudrauf, D. (2015). Preserved emotional awareness of pain in a patient with extensive bilateral damage to the insula, anterior cingulate, and amygdala. Brain Struct Funct.

Gybels, J. M. and Sweet, W. H. (1990). Neurosurgical treatment of persistent pain. Basel, Karger.

Hansen, A. P., Marcussen, N. S., Klit, H., Andersen, G., Finnerup, N. B. and Jensen, T. S. (2012). Pain following stroke: a prospective study. Eur J Pain 16(8): 1128-1136.

Key, B. (2016). Why fish do not feel pain. Animal Sentience 2016.003.

Libet, B. (1973). Electrical stimulation of cortex in human subjects, and conscious sensory aspects. In A. Iggo (Ed.), Handbook of sensory physiology, Vol. II (pp. 743-790). Berlin, Springer-Verlag.

Mazzola, L., Isnard, J. and Mauguière, F. (2006). Somatosensory and pain responses to stimulation of the second somatosensory area (SII) in humans. A comparison with SI and insular responses. Cereb Cortex 16(7): 960-968.

Mazzola, L., Isnard, J., Peyron, R. and Mauguière, F. (2012). Stimulation of the human cortex and the experience of pain: Wilder Penfield's observations revisited. Brain 135(Pt 2): 631-640.

Minert, A. and Devor, M. (2015). Brainstem node for loss of consciousness due to GABA receptor-active anesthetics. Exp Neurol 275P3: 38-45.

Montavont, A., Mauguière, F., Mazzola, L., Garcia-Larrea, L., Catenoix, H., Ryvlin, P. and Isnard, J. (2015). On the origin of painful somatosensory seizures. Neurology 84(6): 594-601. 
Mouraux, A., Diukova, A., Lee, M. C., Wise, R. G. and lannetti, G. D. (2010). A multisensory investigation of the functional significance of the "pain matrix". Neuroimage 54(3): 22372249.

Nair, D. R., Najm, I., Bulacio, J. and Lüders, H. (2001). Painful auras in focal epilepsy. Neurology 57(4): 700-702.

Panksepp, J. (2011). Cross-species affective neuroscience decoding of the primal affective experiences of humans and related animals. PLoS One 6(9): e21236.

Penfield, W. and Faulk, Jr., M. E. (1955). The insula: further observations on its function. Brain 78: $445-470$.

Penfield, W. and Rasmussen, T. (1955). The cerebral cortex of man. New York, MacMillan.

Peyron, R., Laurent, B. and García-Larrea, L. (2000). Functional imaging of brain responses to pain: A review and meta-analysis (2000). Neurophysiol. Clin. 2000: 263-288.

Philippi, C. L., Feinstein, J. S., Khalsa, S. S., Damasio, A., Tranel, D., Landini, G., Williford, K. and Rudrauf, D. (2012). Preserved self-awareness following extensive bilateral brain damage to the insula, anterior cingulate, and medial prefrontal cortices. PLoS One 7(8): e38413.

Posner, J. B., Saper, C. B., Schiff, N. and Plum, F. (2007). Plum and Posner's diagnosis of stupor and coma. New York, Oxford University Press.

Rainville, P., Duncan, G. H., Price, D. D., Carrier, B. and Bushnell, M. C. (1997). Pain affect encoded in human anterior cingulate but not somatosensory cortex. Science 277(5328): 968971.

Roland, P. (1992). Cortical representation of pain. Trends Neurosci 15(1): 3-5.

Starr, C. J., Sawaki, L., Wittenberg, G. F., Burdette, J. H., Oshiro, Y., Quevedo, A. S. and Coghill, R. C. (2009). Roles of the insular cortex in the modulation of pain: insights from brain lesions. J Neurosci 29(9): 2684-2694.

Veldhuijzen, D. S., J. D. Greenspan, J. D., Kim, J. H. and Lenz, F. A. (2010). Altered pain and thermal sensation in subjects with isolated parietal and insular cortical lesions. Eur J Pain 14(5): 535 e531-511.

White, J. and Sweet, W. (1969). Pain and the neurosurgeon. Springfield, Thomas.

Wilkinson, H. A., Davidson, K. M. and Davidson, R. I. (1999). Bilateral anterior cingulotomy for chronic noncancer pain. Neurosurgery 45(5): 1129-1134; discussion 1134-1126. 